\title{
Oxaliplatin-induced sinusoidal obstruction syndrome mimicking metastatic colon cancer in the liver
}

\author{
JUNG-HYE CHOI ${ }^{1}$, YOUNG-WOONG WON ${ }^{1}$, HYUN SUNG KIM ${ }^{2}$, \\ YOUNG-HA OH ${ }^{2}$, SANGHYEOK LIM ${ }^{3}$ and HAN-JOON KIM ${ }^{4}$ \\ Departments of ${ }^{1}$ Internal Medicine, ${ }^{2}$ Pathology, ${ }^{3}$ Radiology and ${ }^{4}$ Surgery, \\ Hanyang University Guri Hospital, Guri, Gyeonggi 471-701, Republic of Korea \\ Received February 21, 2015; Accepted February 11, 2016
}

DOI: $10.3892 / 01.2016 .4286$

\begin{abstract}
Oxaliplatin is an effective chemotherapeutic agent for the treatment of colorectal cancer; however, it may cause liver injury, particularly sinusoidal obstruction syndrome (SOS). Although SOS does not usually present with focal lesions on radiological images, the present study describes the case of a 22-year-old woman with oxaliplatin-induced SOS mimicking metastatic colon cancer in the liver. An abdominal computed tomography revealed a novel $1 \mathrm{~cm}$, low-density lesion in segment 1 of the liver following the administration of the fourth round of oxaliplatin-based adjuvant chemotherapy for stage III colon cancer. Since the lesion was indistinguishable from metastasis, even with detailed imaging studies, including magnetic resonance imaging and positron emission tomography-computed tomography, an isolated caudate lobectomy was planned. The cut surface of the resected liver showed a localized reddish congested lesion measuring $1.4 \mathrm{~cm}$ in diameter. The adjacent hepatic parenchyma also demonstrated diffuse sinusoidal congestion with a nutmeg-like appearance. Histologically, the lesion exhibited severe sinusoidal congestion with peliosis hepatis-like features. The widened sinusoidal space was outlined by markedly attenuated hepatic cords and filled with erythrocytes. The final diagnosis was oxaliplatin-induced SOS. The patient recovered completely and was relapse-free at the time of writing.
\end{abstract}

\section{Introduction}

Fluorouracil (FU)-based adjuvant chemotherapy was the standard treatment in patients with stage III colon cancer until 25 years ago (1). Oxaliplatin has since emerged as an important drug for adjuvant treatment based on clinical

Correspondence to: Dr Jung-Hye Choi, Department of Internal Medicine, Hanyang University Guri Hospital, 153 Gyeongchun, Guri, Gyeonggi 471-701, Republic of Korea

E-mail: jhcmd@hanyang.ac.kr

Key words: oxaliplatin, sinusoidal obstruction syndrome, colon cancer data, as it demonstrates significant activity in metastatic colon cancer. The Multicenter International Study of Oxaliplatin/5-FU/Leucovorin in the Adjuvant Treatment of Colon Cancer trial indicated that adding oxaliplatin to infusional FU plus leucovorin significantly increased disease-free survival and overall survival rates (2). A combination of oxaliplatin and capecitabine is another adjuvant treatment option; therefore, oxaliplatin-based chemotherapy has become popular for the adjuvant treatment of colon cancer (3). Unfortunately, oxaliplatin may cause liver injury, including sinusoidal obstruction syndrome (SOS). SOS may present as reticular hypointensity on hepatobiliary phase images of gadoxetic acid-enhanced magnetic resonance images (EOB-MRI), but does not usually present with focal lesions (4). The present study describes a case of oxaliplatin-induced SOS mimicking metastatic colon cancer in the liver. The lesions were not differentiated from metastasis via imaging and surgery was performed. Written informed consent was obtained from the patient.

\section{Case report}

A 22-year-old woman, who had undergone a laparoscopic right hemicolectomy for ascending colon cancer 4 months prior, presented to Hanyang University Guri Hospital (Guri, South Korea) for the 5th cycle of adjuvant chemotherapy in November 2014. The pathological diagnosis of the ascending colon cancer was stage pT4aN1aM0 moderately-differentiated tubular adenocarcinoma. The patient was treated with oxaliplatin-based adjuvant chemotherapy, consisting of $130 \mathrm{mg} / \mathrm{m}^{2}$ oxaliplatin on day 1 and $1,000 \mathrm{mg} / \mathrm{m}^{2}$ capecitabine twice daily on days 1-14 every 3 weeks (XELOX).

Prior to the fifth cycle of XELOX, the patient exhibited no abnormal signs or symptoms. Laboratory tests revealed a hemoglobin level of $11.8 \mathrm{~g} / \mathrm{dl}$ (normal range, $12.0-16.0 \mathrm{~g} / \mathrm{dl}$ ), a white blood cell count of $3,800 \mathrm{cells} / \mu \mathrm{l}$ (normal range, 4,000-10,000 cells $/ \mu 1$ ), a platelet count of 112,000 platelets $/ \mu 1$ (normal range, $150,000-400,000$ platelets/ $\mu 1$ ), total bilirubin level of $0.6 \mathrm{~g} / \mathrm{dl}$ (normal range, 0.4-1.5 g/dl), alkaline phosphatase level of 105 IU/1 (normal range, 35-95 IU/1), alanine aminotransferase concentration of 51 IU/1 (normal range, 10-45 IU/1), aspartate aminotransferase concentration of 47 IU/1 (normal range, 15-45 IU/l), and normal results 
A

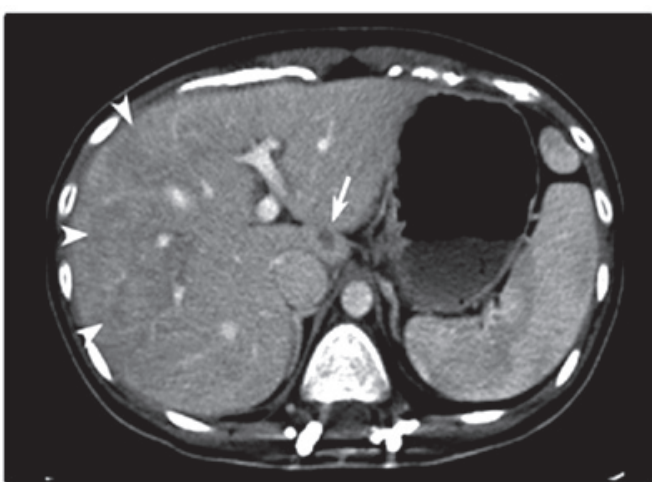

C

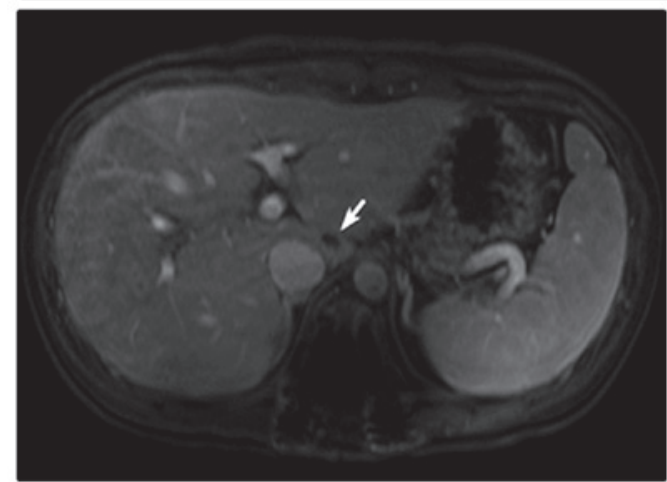

$\mathbf{E}$

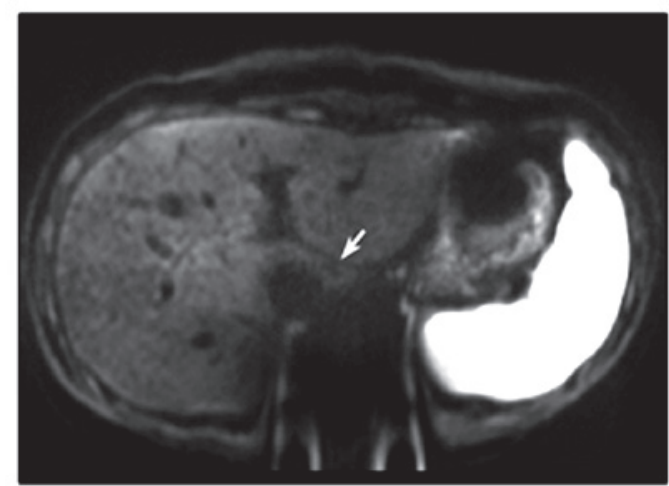

B

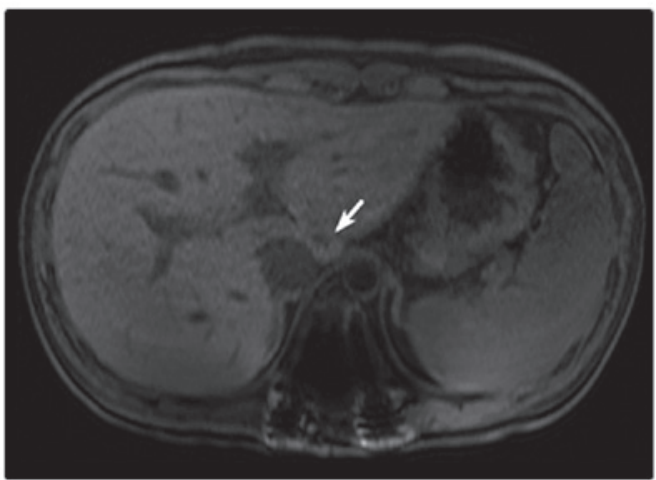

D

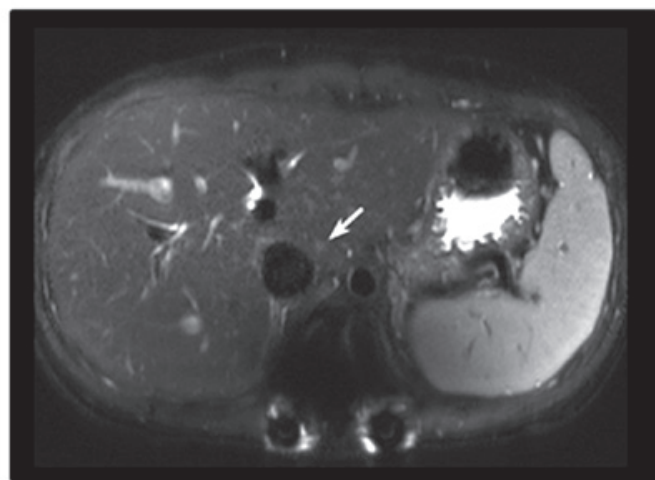

$\mathbf{F}$

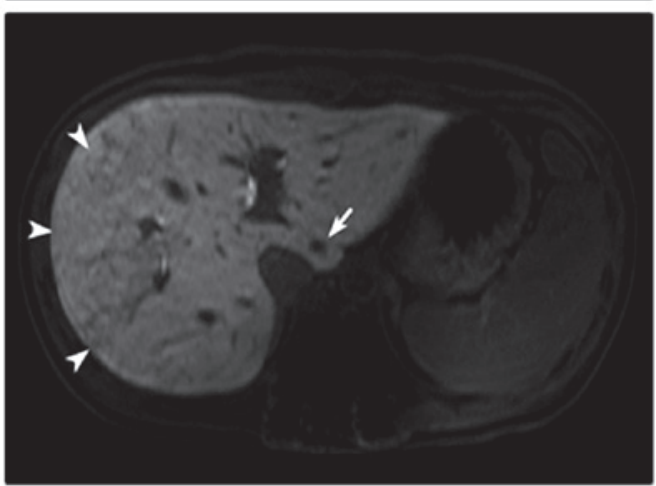

Figure 1. Imaging studies of a 22-year-old woman with a single, newly developed, $1.0 \mathrm{~cm}$ lesion in segment 1 of the liver, detected 3 months subsequent to the initiation of oxaliplatin-based adjuvant chemotherapy for colon cancer. (A) Transverse contrast-enhanced computed tomography images revealed a nodular low-density lesion (arrow) and poorly enhanced areas (arrowheads). On the EOB-MRI images, the lesion (arrows) was hypointense in the (B) pre-contrast and (C) portal phase T1-weighted images without enhancement, (D) partially hyperintense on the T2-weighted images, and (E) hypointense on the diffusion-weighted images $\left(b=800 \mathrm{sec} / \mathrm{mm}^{2}\right.$. . F EOB-MRI images revealed reticular hypointensity on the hepatobiliary phase images in the right lobe of the liver (arrowheads). EOB-MRI, gadoxetic acid-enhanced magnetic resonance imaging.

for kidney function tests. The carcinoembryonic antigen was within the normal range $(3.94 \mathrm{ng} / \mathrm{ml}$; normal range, 0.1-5.0 $\mathrm{ng} / \mathrm{ml}$ ), and the results of the serological tests revealed that the patient was not infected with hepatitis $\mathrm{B}$ or $\mathrm{C}$ virus.

The contrast-enhanced abdominal computed tomography (CT) scan revealed a novel tiny, nodular, low-density lesion in segment 1 of the liver. EOB-MRI identified that the lesion was $1 \mathrm{~cm}$ in size at the widest point, ovoid and hypointense on the pre-contrast and portal-phase T1-weighted imaging, without enhancement. EOB-MRI revealed partial hyperintensity on the T2-weighted imaging and a hypointense nodule on diffusion-weighted imaging (Fig. 1). Positron emission tomography (PET)-CT identified no clear abnormal fluorodeoxyglucose (FDG) uptake suggestive of malignancy. Although the findings did not exclude a metastatic lesion, adjuvant chemotherapy [oxaliplatin (190 mg, day 1) and capecitabine $(1,500 \mathrm{mg}$, twice daily, days $1-14)$ every
3 weeks] was administered, with a follow-up abdominal CT performed one month later.

Abdominal CT following the fifth round of chemotherapy revealed that the lesion was more discrete and slightly enlarged. Hepatobiliary ultrasonography revealed a $1.4-\mathrm{cm}$ mixed echoic lesion in segment 1 and mild splenomegaly $(12.3 \mathrm{~cm}$; prior to chemotherapy, $11 \mathrm{~cm})$. Due to the changes observed in the lesion, a surgical resection was planned.

The patient underwent isolated caudate lobectomy of the liver. The liver was exposed through an inverted $\mathrm{T}$ incision. Following the cholecystectomy, the falciform ligament was divided and a liver mobilization was performed. A tumor was not visible or palpable upon gross observation of the caudate lobe. Intraoperative ultrasonography revealed a $1.5-\mathrm{cm}$ lesion in the caudate lobe. The adjacent liver parenchyma was normal. For the isolated caudate lobectomy, the hanging maneuver was applied (5). 

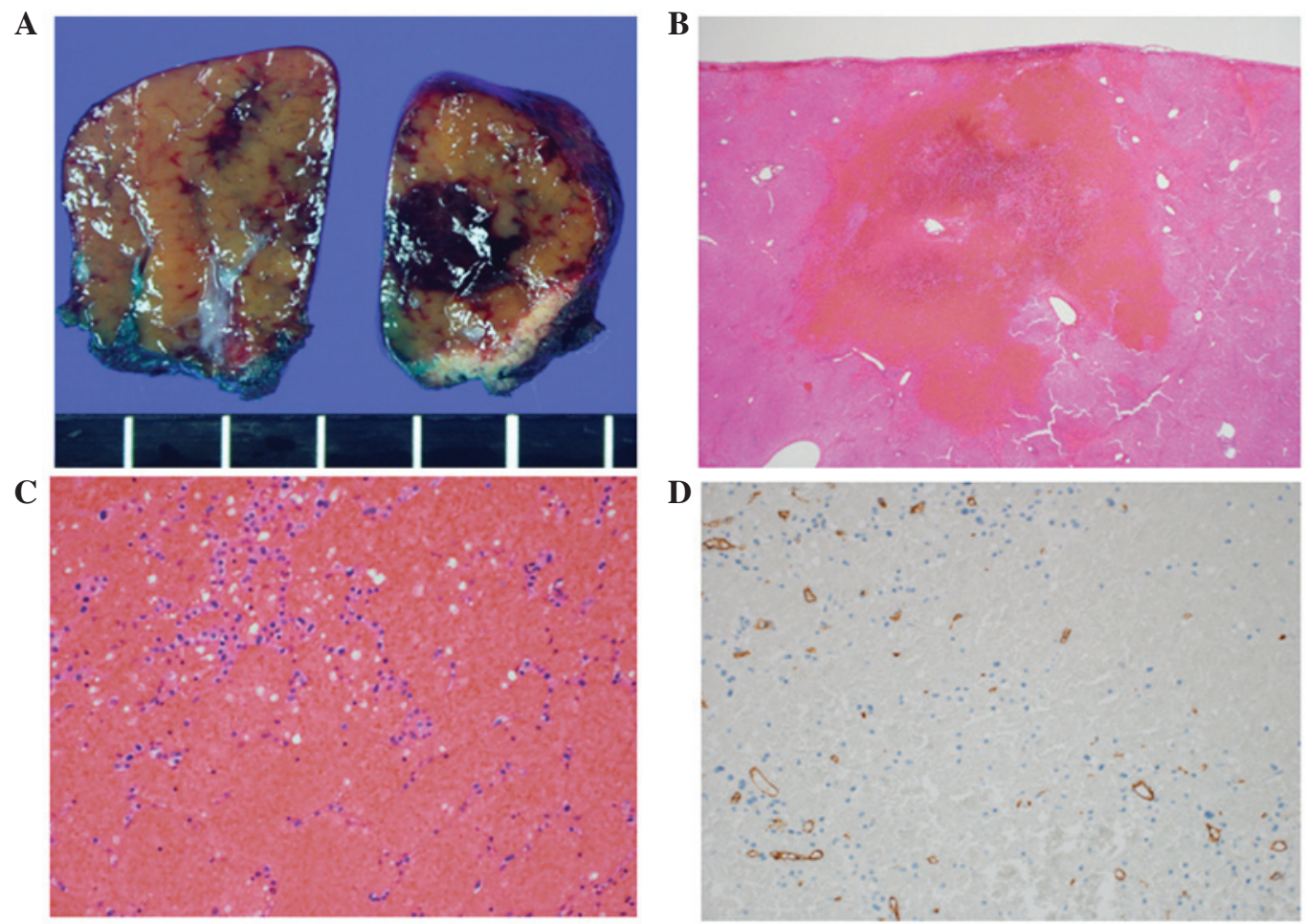

Figure 2. (A) Macroscopic cross-sections revealed a 1.4x1.0 cm, ill-defined, dark-red congested lesion in the subcapsular area. The adjacent parenchyma also revealed sparsely distributed dark-red congestion. Microscopically, the lesion revealed severe sinusoidal dilation outlined by atrophic hepatocytes, as shoen by hematoxylin and eosin staining at a magnification of (B) x12.5 and (C) x200. (D) Cluster of differentiation 34 immunohistochemical staining detected decreased sinusoidal endothelial cells (magnification, $\mathrm{x} 200$ ).

Macroscopically, an ill-defined, dark, red, congested lesion with a soft consistency measuring $1.4 \times 1.0 \mathrm{~cm}$ on the cross-section was identified in the resected caudate lobe of the liver. The lesion identified was considered to be the same lesion that had indicated malignancy on the radiological studies. The adjacent parenchyma also demonstrated diffuse sinusoidal congestion and had a nutmeg-like appearance (Fig. 2A). Microscopically, the lesion exhibited severe sinusoidal dilation with congestion. The widened sinusoidal space was outlined by markedly attenuated hepatic cords and filled with erythrocytes. An immunohistochemical study of cluster of differentiation 34 revealed a decrease in sinusoidal endothelial cells in this lesion (Fig. 2B-2D). The final diagnosis was oxaliplatin-induced SOS.

The patient had an uneventful post-operative course and recovered completely. Since the SOS was revealed histologically in the lesion and the surrounding liver tissue, administration of XELOX was discontinued. The patient remained relapse-free for three months following the lobectomy.

\section{Discussion}

Oxaliplatin, a third-generation platinum analog, is an effective chemotherapeutic agent for numerous solid tumors when combined with other drugs, including colorectal and stomach cancer. Since the first clinical study that demonstrated severe hepatic sinusoidal obstruction induced by oxaliplatin in 2004 (6), oxaliplatin-induced hepatic injury has become a major concern in patients that receive hepatic resection for metastatic colorectal cancer. Oxaliplatin-induced hepatic injury often manifests as SOS. SOS, previously termed veno-occlusive disease or blue liver syndrome, is characterized by the discontinuity of the sinusoidal membrane, collagenization of the perisinusoidal space and sinusoidal dilatations with erythrocyte congestion in centrilobular zones due to damage to the sinusoidal endothelial cells (6-8).

The majority of oxaliplatin-induced SOS data are obtained by reviewing post-chemotherapy liver resection specimens obtained from patients with colorectal liver metastases that received preoperative chemotherapy $(6,8,9)$. The patient in the present study developed a hepatic lesion during adjuvant chemotherapy. The lesion was not differentiated from metastasis despite the use of several imaging tools. Although the hepatic lesion demonstrated no abnormal FDG uptake on the PET-CT and no abnormalities on the diffusion-restriction MRI, the lesion had increased in size one month later. Due to this finding, the possibility of metastasis was considered first. A biopsy of the lesion was initially planned; however, due to the risk of complications from the biopsy and, if the lesion was metastatic, a lobectomy was performed without a biopsy.

To the best of our knowledge, there have previously only been two reported cases of SOS mimicking a metastatic tumor on imaging that are similar to the present study $(10,11)$. The two previous studies described patients that had developed several novel hepatic lesions during adjuvant oxaliplatin-based chemotherapy, including FOLFOX6, which consists of oxaliplatin with FU and folinic acid, and XELOX $(10,11)$. These previous studies did not consider the possibility of non-malignant lesions prior to performing the lobectomy. 
Although oxaliplatin-induced SOS is usually asymptomatic, it may be associated with an increased perioperative morbidity and bleeding risk (9). Therefore, there have been several studies that investigated the predictive parameters for oxaliplatin-induced SOS, such as EOB-MRI findings, the volume of the spleen, the levels of hyaluronic acid, the indocyanine green retention rate at $15 \mathrm{~min}$ (ICG-R15), and aspartate aminotransferase (AST) level $(8,9,12)$. Shin et al (4) reported that reticular hypointensity on hepatobiliary phase images of EOB-MRI was highly specific for SOS. Shin et al divided the presence of reticular hypointensity into 5 levels, and levels 4 and 5 were considered to indicate SOS (4). In the present study, transverse contrast-enhanced CT imaging revealed diffuse, poorly enhanced regions on the right lobe of the liver, and EOB-MRI revealed confidence level 4 reticular hypointensity on the hepatobiliary phase images in the same region (Fig. 1F). In addition, the AST levels (54 IU/1) and ICG-R15 (12.25\%) were increased prior to surgery. The findings suggested a diagnosis of oxaliplatin-induced SOS in the background liver. However, during the initial evaluation, only the focal lesion in segment 1 was focused on, making the diagnosis of SOS prior to surgery extremely challenging.

In summary, the present study reports the case of a patient with oxaliplatin-induced SOS that mimicked metastatic colon cancer in the liver on imaging studies. Therefore, SOS may be considered one of the causes of newly developed hepatic lesions in patients with colon cancer that receive oxaliplatin-based chemotherapy, particularly if the patients possess predictive findings for oxaliplatin-induced SOS and the lesions do not demonstrate the uptake of FDG on the PET-CT.

\section{References}

1. Moertel CG, Fleming TR, Macdonald JS, Haller DG, Laurie JA, Goodman PJ, Ungerleider JS, Emerson WA, Tormey DC, Glick JH, et al: Levamisole and fluorouracil for adjuvant therapy of resected colon carcinoma. N Engl J Med 322: 352-358, 1990.
2. André T, Boni C, Navarro M, Tabernero J, Hickish T, Topham C, Bonetti A, Clingan P, Bridgewater J, Rivera F and de Gramont A: Improved overall survival with oxaliplatin, fluorouracil, and leucovorin as adjuvant treatment in stage II or III colon cancer in the MOSAIC trial. J Clin Oncol 27: 3109-3116, 2009.

3. Haller DG, Tabernero J, Maroun J, de Braud F, Price T, Van Cutsem E, Hill M, Gilberg F, Rittweger K and Schmoll HJ: Capecitabine plus oxaliplatin compared with fluorouracil and folinic acid as adjuvant therapy for stage III colon cancer. J Clin Oncol 29: 1465-1471, 2011.

4. Shin NY, Kim MJ, Lim JS, Park MS, Chung YE, Choi JY, Kim KW and Park YN: Accuracy of gadoxetic acid-enhanced magnetic resonance imaging for the diagnosis of sinusoidal obstruction syndrome in patients with chemotherapy-treated colorectal liver metastases. Eur Radiol 22: 864-871, 2012.

5. Kim SH, Park SJ, Lee SA, Lee WJ, Park JW and Kim CM: Isolated caudate lobectomy using the hanging maneuver. Surgery 139: 847-850, 2006.

6. Rubbia-Brandt L, Audard V, Sartoretti P, Roth AD, Brezault C, Le Charpentier M, Dousset B, Morel P, Soubrane O, Chaussade S, et al: Severe hepatic sinusoidal obstruction associated with oxaliplatin-based chemotherapy in patients with metastatic colorectal cancer. Ann Oncol 15: 460-466, 2004.

7. DeLeve LD, Shulman HM and McDonald GB: Toxic injury to hepatic sinusoids: Sinusoidal obstruction syndrome (veno-occlusive disease). Semin Liver Dis 22: 27-42, 2002.

8. Morine Y, Shimada M and Utsunomiya T: Evaluation and management of hepatic injury induced by oxaliplatin-based chemotherapy in patients with hepatic resection for colorectal liver metastasis. Hepatol Res 44: 59-69, 2014

9. Aloia T, Sebagh M, Plasse M, Karam V, Lévi F, Giacchetti S, Azoulay D, Bismuth H, Castaing D and Adam R: Liver histology and surgical outcomes after preoperative chemotherapy with fluorouracil plus oxaliplatin in colorectal cancer liver metastases. J Clin Oncol 24: 4983-4990, 2006.

10. Arakawa Y, Shimada M, Utsunomya T, Imura S, Morine Y, Ikemoto T, Hanaoka J, Sugimoto K and Bando Y: Oxaliplatin-related sinusoidal obstruction syndrome mimicking metastatic liver tumors. Hepatol Res 43: 685-689, 2013.

11. Uchino K, Fujisawa M, Watanabe T, Endo Y, Nobuhisa T, Matsumoto Y, Kai K, Sato S, Notohara K and Matsukawa A: Oxaliplatin-induced liver injury mimicking metastatic tumor on images: A case report. Jpn J Clin Oncol 43: 1034-1038, 2013.

12. van den Broek MA, Vreuls CP, Winstanley A, Jansen RL, van Bijnen AA, Dello SA, Bemelmans MH, Dejong $\mathrm{CH}$, Driessen A and Olde Damink SW: Hyaluronic acid as a marker of hepatic sinusoidal obstruction syndrome secondary to oxaliplatin-based chemotherapy in patients with colorectal liver metastases. Ann Surg Oncol 20: 1462-1469, 2013. 